\title{
«Le difficoltà del comprendere»: la Shoah come genocidio, tra storia e microstoria
}

\author{
di Simon Levis Sullam
}

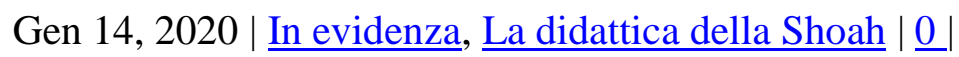

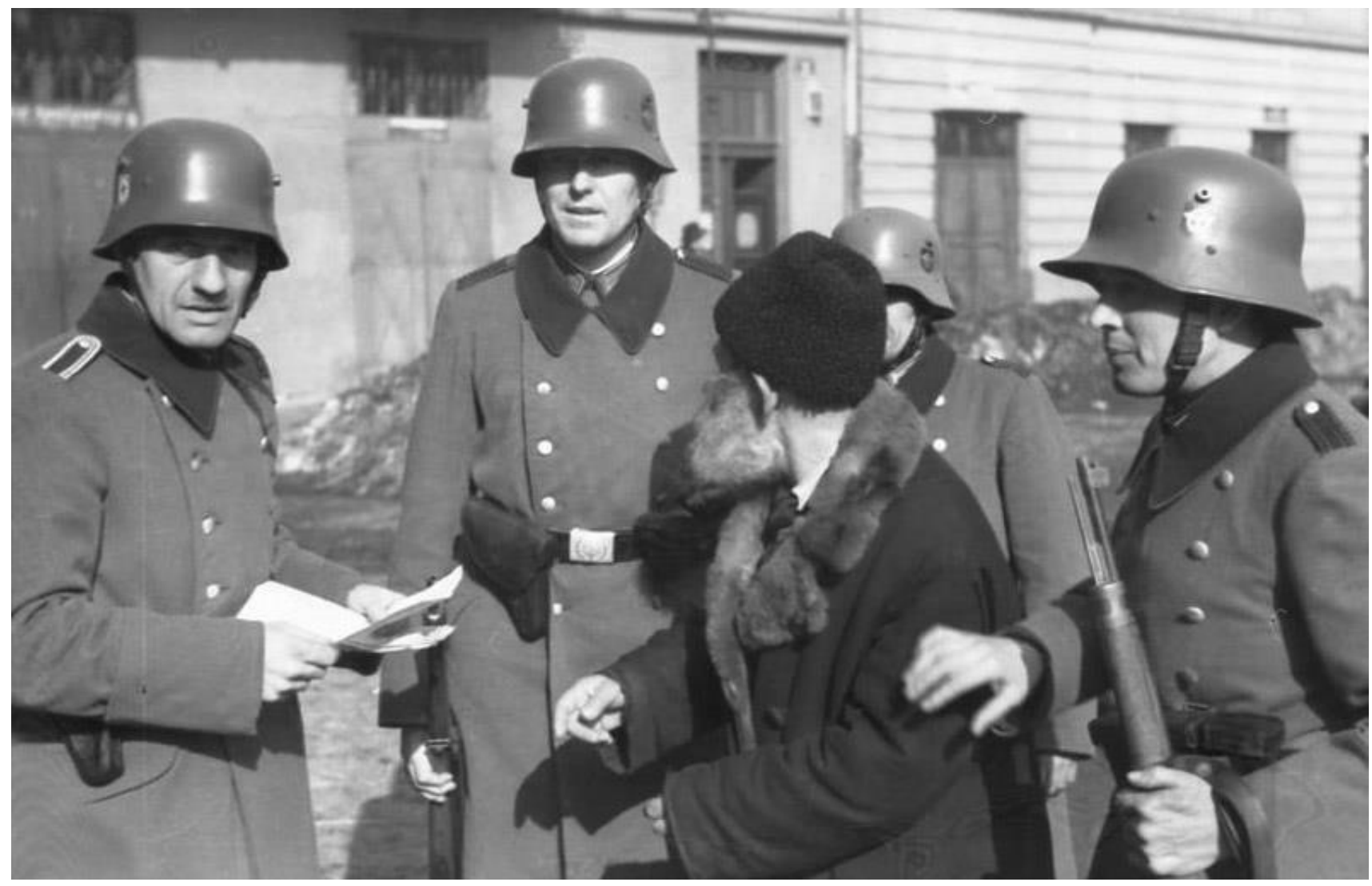

Cracovia (Polonia): razzia da parte della Ordnungspolizei nel 1941.

By Bundesarchiv, Bild 101I-030-0780-28 / Kintscher / CC-BY-SA 3.0, CC BY-SA 3.0 de, Link

\begin{abstract}
L'intervento passa in rassegna alcuni studi sulla storia della Shoah negli ultimi venticinque anni, in cui è crescentemente emerso un approccio microstorico alle vicende della «Soluzione finale».

Questi studi hanno affrontato il problema della ricostruzione del punto di vista (ad es. dei carnefici o delle vittime), hanno messo a fuoco la natura e le trasformazioni dei rapporti umani nei genocidi; hanno infine tentato di interpretare la natura allo stesso tempo «ordinaria» e «criminale» della violenza nello sterminio.
\end{abstract}

«Le difficoltà del comprendere»: la Shoah come genocidio, tra storia e microstoria

La produzione storiografica sullo sterminio degli ebrei nell'ultimo quarto di secolo è stata segnata dalla comparsa di alcune opere che hanno offerto uno sguardo macrostorico e, crescentemente, microstorico alle vicende del nazismo, della persecuzione degli ebrei e della Seconda guerra mondiale. Sebbene in modo non esclusivo la categoria di «genocidio» si è dimostrata rilevante nelle nuove interpretazioni di quelle vicende, consentendo di superare una prospettiva che insisteva 
sull'«unicità» della Shoah, di stabilire quindi delle comparazioni con altri stermini ed episodi di violenza estrema nella storia e di ricorrere a spiegazioni complesse delle origini e degli eventi dell'Olocausto. Spiegazioni che andassero oltre quelle monocausali, focalizzate in particolare sull'antisemitismo come motivazione ideologica centrale dei persecutori.

\section{La categoria di «genocidio»}

Com'è noto la categoria di «genocidio» venne definita nel corso stesso della Seconda guerra mondiale dal giurista ebreo polacco Raphael Lemkin come «il tentativo di distruggere, in tutto o in parte, un gruppo nazionale, etnico, razziale o religioso, come tale». Lemkin si riferiva sia agli eventi in corso che al genocidio degli Armeni (1915-1917) e , alcuni anni dopo, la sua definizione verrà posta alla base della Convenzione per la prevenzione e la repressione del delitto di genocidio, adottata dall'ONU nel dicembre 1948[1]. L'utilizzo storiografico della categoria di genocidio si è affermato particolarmente negli anni 2000 (pensiamo ad esempio a Donald Bloxham, Lo sterminio degli ebrei. Un genocidio[2]), mentre in precedenti stagioni della storiografia altre categorie erano prevalse: basterà ricordare ad esempio i titoli del pionieristico Léon Poliakov, Il nazismo e lo sterminio degli ebrei[3], o del classico Raul Hilberg, La distruzione degli ebrei d'Europa[4]. Il vocabolo «olocausto» si è invece affermato negli anni Ottanta del Novecento sulla scia della serie televisiva Holocaust, e gradualmente è divenuto termine relativamente neutro - nonostante la sua etimologia religiosa e sacrificale - almeno nella lingua inglese: pensiamo alla stessa categoria degli «Holocaust studies». Shoah, che in ebraico significa «catastrofe», si diffonde negli anni Novanta del secolo scorso: pur essendo oggi probabilmente il più diffuso almeno nel contesto del discorso pubblico italiano, ha il limite di segnare una sorta di primato o esclusivismo ebraico sulle vicende della «Soluzione finale»[5]. Assieme allo hilbergiano «distruzione», il lemma «genocidio» degli ebrei ci pare oggi il più neutro ed efficace in ambito storiografico.

\section{Una rassegna di studi}

Gli studi che passeremo in sintetica rassegna qui hanno assunto diverse scale di osservazione delle vicende della Shoah - macro e micro - e si sono confrontate con il problema del punto di vista adottato nella ricostruzione storica: hanno affrontato le «difficoltà del comprendere» (secondo l'espressione di Hannah Arendt a proposito del totalitarismo) relazionandosi in modo diverso con lo sguardo dei carnefici e delle vittime; hanno inoltre messo a fuoco la natura e le trasformazioni dei rapporti umani nei genocidi; hanno infine tentato di interpretare la natura allo stesso tempo «ordinaria» e «criminale» della violenza della «Soluzione finale». In questi ambiti sono emersi alcuni dei principali nodi epistemologici della più recente storiografia della Shoah e le soluzioni metodologiche che essa ha suggerito.

\section{L'analisi di Saul Friedländer}

L'analisi proposta da uno dei decani della storiografia della Shoah, Saul Friedländer, nel suo Gli anni dello sterminio[6], muove dalla focalizzazione germano-centrica che aveva caratterizzato il suo precedente volume del 1997, La Germania nazista e gli ebrei (1933-1938)[7], verso una prospettiva globale su scala europea. Restano centrali in questa disamina la dimensione culturale, politica ed ideologica con una fondamentale rilevanza del fattore del cosiddetto «antisemitismo redentivo». Estremamente rilevante in questa ricostruzione è il ruolo di Hitler, come una sorta di 
«deus ex machina»; ma non viene trascurato ad esempio l'atteggiamento connivente, ricostruito con attenzione, delle Chiese cristiane come attori-spettatori comprimari. L'analisi di Friedländer, particolarmente concentrata sul livello individuale dell'esperienza, dell'azione e dell'osservazione, definito «microlivello», alterna costantemente la prospettiva dei carnefici (dai diari di Goebbels alle lettere dei soldati sul fronte orientale) con quella delle vittime, soprattutto attraverso diari, lettere e testimonianze, attribuendo a quest'ultima una funzione fondamentale nella narrazione. Anche in una ricostruzione che guarda all'insieme degli eventi tra l'autunno 1939 e la primavera del 1945 nell'intero teatro del continente europeo, lo sguardo singolo, individuale, del microlivello - a cui avviene l'interazione fondamentale e continuativa degli ebrei con le forze che agiscono nell'implementazione della «Soluzione finale» - rappresenta il punto di osservazione fondamentale delle vicende ricostruite. Friedländer sottolinea come, se lo scopo della conoscenza storica è addomesticare l'incredulità, il suo scopo è stato quello di ricostruire lo sterminio degli ebrei senza eliminare quel senso di incredulità: quello dei protagonisti e testimoni diretti degli eventi di fronte al loro svolgersi; e quello di chi legge o ascolta, per così dire assiste alle ricostruzioni storiche successive[8]. Come Friedländer scrive già nel primo volume della sua opera, quelle delle vittime «sono le sole voci in cui viene a fondersi la più lucida presa di coscienza e la totale cecità di esseri umani posti innanzi a una realtà del tutto nuova e assolutamente terrificante». Lo storico si dice interessato a rappresentare «la natura assolutamente criminale e al contempo assolutamente ordinaria» degli eventi della persecuzione e dello sterminio: lo ha fatto «contrapponendo livelli di realtà del tutto diversi - ad esempio, dibattiti ad alto livello sulla politica antiebraica [...] a comuni scene di persecuzione - al fine di creare un senso di estraneazione che si contrapponga alla nostra tendenza ad 'assuefarci' a quel particolare passato». "Tale senso di estraniazione», aggiunge Friedländer, riflette «la percezione delle vittime [...] di una realtà assurda e minacciosa, di un mondo al contempo grottesco e agghiacciante sotto una patina di ancor più agghiacciante normalità»[9].

\section{L’approccio microstorico di Jan T. Gross}

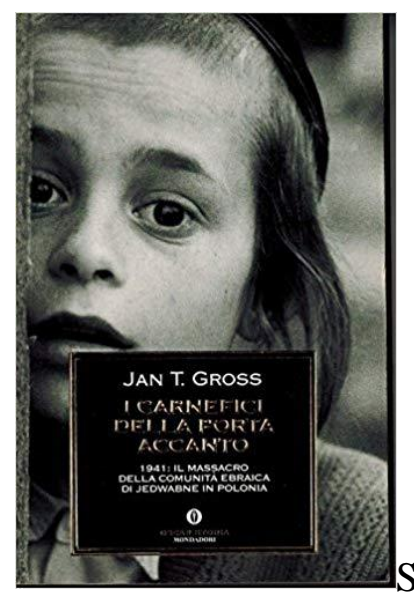

2001 di Jan T. Gross, dedicato al massacro della comunità ebraica di Jedwabne in Polonia, con il titolo I carnefici della porta accanto, ad aver portato alla ribalta l'approccio microstorico. L'opera è incentrata sul racconto delle vicende di un'unica giornata, il 10 luglio 1941, in cui metà del villaggio di Jedwabne massacrò improvvisamente l'altra metà, costituita da circa milleseicento ebrei. Il villaggio era occupato dai nazisti, ma l'iniziativa e l'azione fu degli abitanti polacchi. La vicenda è ricostruita sulla base di fonti storiche e soprattutto giudiziarie del dopoguerra e mette in luce il ruolo e le responsabilità dei polacchi in quanto «uomini comuni» (secondo l'espressione di Christopher Browning), motivati dal pregiudizio antiebraico, ma anche dalla volontà di 
arricchimento, che fecero da leva all'odio scoppiato all'interno di una piccola comunità. Come nota Gross:

Quelli che gli ebrei si trovarono con orrore e, oserei dire, con stupore di fronte furono volti familiari. Non anonimi individui in divisa, ingranaggi di una macchina da guerra, agenti che eseguivano gli ordini, ma vicini di casa, quelli della porta accanto, che scelsero di uccidere e presero parte a un sanguinoso pogrom come volenterosi carnefici[10].

Questa constatazione mette in luce come il genocidio non sia opera di soggetti stranieri, che operano in terre lontane, ma abbia spesso le caratteristiche di una violenza fratricida che si svolge all'interno delle società, condotta dai loro stessi abitanti. Molteplici furono le motivazioni all'origine del massacro, non riconducibili al solo antisemitismo. Prendendovi parte, nota infatti Gross, gli abitanti di Jedwabne potevano

ingraziarsi i nuovi dominatori [nazisti], trarre benefici materiali dalle proprie azioni [...] $e$ dare sfogo alla tradizionale ostilità dei contadini locali verso gli ebrei. Se aggiungiamo l'incoraggiamento dei nazisti e l'impressione [...] che si trattava di regolare $i$ conti con la 'consorteria giudaica' per le angherie subite durante l'occupazione sovietica, chi poteva resistere a una miscela tanto diabolica ed esplosiva?[11].

A tutto ciò si aggiungeva «una brutalizzazione delle relazioni interpersonali, una certa caduta dei valori morali e una generale autorizzazione all'uso della violenza»[12], cioè fattori - potremmo aggiungere - sempre presenti come presupposti nel contesto dei genocidi. Queste caratteristiche della vicenda ne fanno sì un «caso limite», ma allo stesso tempo gettano luce su alcuni aspetti più generali dello sterminio degli ebrei e delle motivazioni dei persecutori. Com'è tipico dell'approccio microstorico la descrizione intensiva di un caso locale e la messa in luce dei fattori causanti e delle implicazioni più ampie fanno apparire la vicenda di Jedwabne come «eccezionale normale», secondo la definizione che lo storico Edoardo Grendi ha dato dei casi oggetto tipico di studio da parte della microstoria. Si tratta di casi in cui le caratteristiche di vicende e contesti storici emergono con particolare intensità, in modo spiccato, tanto da apparire straordinari, ma si rivelano in realtà rappresentativi di aspetti e dinamiche più generali e ordinarie[13]. La descrizione densa delle vicende e degli attori - in una sorta di «biografia collettiva»[14] - in un unico giorno e in un unico luogo di un episodio sanguinoso della storia del genocidio degli ebrei fa emergere caratteristiche ricorrenti e strutturali, che si ritrovano in altri casi o segnano fattori e avvenimenti che si ripetono, rappresentando con particolare vividezza la natura del fenomeno storico della Shoah.

\section{Timothy Snyder}

Gli stessi territori e alcune delle medesime dinamiche di cui si occupa Gross, sono al centro di un'altra opera che utilizza un approccio macrostorico alle vicende dello sterminio degli ebrei e ai massacri della Seconda guerra mondiale, utilizzando una cronologia più lunga che include anche le violenze degli anni Trenta. Si tratta del volume di Timothy Snyder, Terre di sangue. L'Europa nella morsa di Hitler e Stalin, apparso in italiano nel 2011[15]. Snyder mette al centro della sua ricostruzione - una sorta di analisi geopolitica dall'alto - una vasta area geografica che include Polonia, Ucrania, Bielorussia, Lituania, Lettonia, Estonia: le cosiddette «terre di sangue», oggetto di occupazione successiva da parte di sovietici e nazisti. Risale inoltre cronologicamente all'indietro alle vicende della Grande carestia (1932-33) e del Grande terrore (1936-1938) sovietici individuandoli tra le matrici di un'epoca di violenze collettive che fino al 1945 produsse 
complessivamente 14 milioni di morti. Se verso la fine del 1938 anche le violenze naziste in Germania assunsero particolare intesità, Snyder ritiene che in quell'anno la violenza etnica e antisemita sovietica superasse quella nazista. Inoltre sottolinea gli effetti nel corso della guerra mondiale delle invasioni successive di nazisti e sovietici, in cui spesso la violenza nazista rappresentò - o venne presentata come - una ritorsione agli effetti dell'occupazione sovietica. Così, pur senza citarlo, Snyder riprende in modo controverso la discussa interpretazione di Ernest Nolte, nel suo La guerra civile europea[16], che aveva indicato nelle violenze bolsceviche il precedente storico e logico di quelle naziste. In questo caso l'autore spiega perché abbia preferito il termine «sterminio» a «genocidio»[17], in quanto - con riferimento alla Grande fame e al Grande terrore l'«assassinio di massa» fu privo di componenti o motivazioni etniche immediatamente evidenti e fu motivato dagli ideatori dei massacri piuttosto sul piano economico e politico. In realtà egli ricorda come lo stesso Lemkin avesse definito la carestia in Ucraina come genocidio. Nonostante l'analisi macroscopica di Snyder non si soffermi sulle vicende minute delle violenze, cioè non si spinga ad assumere o descrivere in dettaglio i punti di vista degli attori implicati, egli insiste sulla necessità per lo storico di identificarsi innanzitutto con i carnefici: «È meno allettante, ma più urgente da un punto di vista morale», scrive, «capire le azioni dei colpevoli. Dopotutto il pericolo morale non è mai costituito dalla possibilità di diventare una vittima, ma di trasformarsi in un persecutore o uno spettatore». «La tentazione», mette in guardia inoltre Snyder, «di affermare che un assassino nazista si colloca oltre i limiti della comprensione è concreta»[18].

\section{Omer Bartov}

Torniamo alla prospettiva microstorica - e alla centralità della categoria di «genocidio» - con lo studio di Omer Bartov, Anatomy of a Genocide. The Life and Death of a Town called Buczacz [19]. Anche in questo caso si tratta della descrizione ed analisi delle violenze in un'unica cittadina ucraina, quella di Buczacz, studiata in un lungo periodo che risale fino alla Prima guerra mondiale. Bartov esamina, attraverso la descrizione densa di un territorio circoscritto, le relazioni politiche e sociali, i rapporti di forza degli attori innanzitutto locali, in una prospettiva che alterna la ricostruzione di singoli eventi a un'analisi dei fenomeni di lunga durata. Al centro della narrazione si collocano tre gruppi etnici: polacchi, ebrei ed ucraini; e tre successivi occupanti: austriaci, sovietici e nazisti. Questi diversi attori producono in momenti diversi atteggiamenti ed azioni di violenza e di solidarietà, in particolare nei confronti della minoranza ebraica. Il processo analizzato da Bartov fin dai prodromi del primo conflitto mondiale vede i tre gruppi etnici crescentemente

chiudersi in se stessi non solo come in passato ignorandosi a vicenda, ma in modo più aggressivo, risentito e accusatorio, percependo le proprie difficoltà come conseguenza della condotta dell'altro e considerando i diritti degli altri come limitanti i propri[20].

Una situazione non ideale per una futura coesistenza pacifica. L'autore segue in effetti la crescente «logica della separazione tra vicini», sebbene il nemico prevalente resti l'ebreo, fino allo scoppio feroce del «conflitto fratricida»[21]. A Bartov dobbiamo tra i primi la messa a fuoco della rilevanza degli spazi est-europei per lo studio della violenza genocida[22] e in particolare di quella che ha chiamato l'«intimità della violenza» tra vicini[23]. In quest'opera lo storico israeliano dimostra come attraverso le situazioni delle successive occupazioni sovietiche e nazista, polacchi, ucraini ed ebrei «videro se stessi come le vittime principali delle diverse configurazioni dell'occupazione sovietica e tedesca» e come «ciascuno percepì la persecuzione degli altri due gruppi come almeno in parte giustificata». «La convinzione di ogni gruppo dell'unicità della propria vittimizzazione si accompagnò con un desiderio di punire coloro che erano associati con la propria sofferenza»[24]. Bartov si sofferma particolarmente sulla natura quotidiana della violenza e su come essa si intrecciò 
con il tessuto della vita cittadina; riflette sul «mix surreale di orrore e normalità»[25] che emerge anche dalle testimonianze del dopoguerra a proposito delle vicende di Buczaz. In queste si delinea «la natura pubblica e disinvolta delle uccisioni e il senso di impunità e onnipotenza dei perpetratori, il loro assoluto potere sulla vita e la morte». Secondo una testimone oculare, durante i massacri, «gli ebrei venivano cacciati sulle strade come conigli. Ebrei in fuga venivano giustiziati sul posto»; la testimone ricordava di averne visti cadere a decine con pallottole sparate alla nuca in una fossa scavata nel locale cimitero ebraico. Un giorno uno dei responsabili dei massacri si presentò dicendo: «Oggi ho ucciso il mio milleduecentesimo ebreo»[26].

\section{Christopher Browning e la natura «ordinaria» del genocidio}

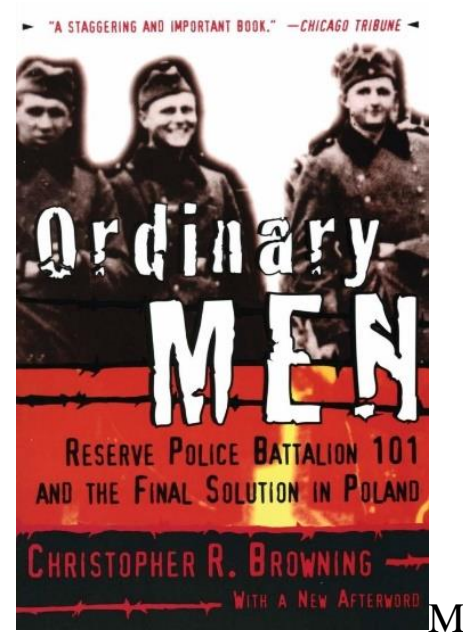

Ma il capostipite di queste ricostruzioni microstoriche e lo studioso che per primo ha messo in luce la natura «ordinaria» del genocidio è stato Christopher Browning con la sua opera Uomini comuni. Polizia tedesca e «soluzione finale» in Polonia [27]. Browning presenta esplicitamente il suo lavoro come dedicato a un «caso di studio o microstoria di un singolo battaglione», basato sulla «descrizione densa» che egli riferisce all'approccio storiografico della «storia della vita quotidiana» tedesca (Alltagsgeschichte)[28]. Com'è ben noto prende in esame sulla base di procedimenti giudiziari del dopoguerra le vicende del Battaglione 101 della Riserva di Polizia tedesca. Si tratta di un gruppo di uomini non ideologicamente motivati, ma operai, impiegati, commercianti, artigiani che si ritrovarono coinvolti nella «Soluzione finale»e giunsero a sterminare 1500 persone in un giorno nel villaggio polacco di Jozefow. Secondo la ricostruzione di Browning, a questi uomini fu data in principio la possibilità di rinunciare al loro compito, di non partecipare al massacro, ma quasi nessuno rinunciò. Lo storico ci mostra che nell'impegno in uccisioni seriali e nell'assuefazione alla violenza i protagonisti del suo racconto non erano «tedeschi» comuni, ma «uomini» comuni: nessuna motivazione ideologica, né il patriottismo, né tantomeno una particolare inclinazione nazionale motivò le loro azioni. Essi furono condizionati dal rispetto degli ordini, dalla pressione dei commilitoni, dallo spirito di gruppo, dal clima della guerra e dalla caduta di ogni tabù morale - ma anche dallo spirito di emulazione e dal desiderio di carriera - in un contesto di partecipazione collettiva. Come nota Browning, «in ultima analisi, l'Olocausto fu possibile perché singoli esseri umani uccisero altri esseri umani in gran numero e per un lungo periodo di tempo. I semplici esecutori divennero 'assassini di professione'»[29]. Sul piano metodologico Browning si sofferma particolarmente sulla questione del punto di vista dello storico, assumendo un atteggiamento di «immedesimazione» anche quando egli descrive «dal basso» le più crude scene di «face-to-face killing», le fucilazioni di massa di fronte alle fosse comuni. Nota in proposito lo storico americano 
$\grave{E}$ evidente che scrivere una storia di questo tipo significa rifiutare la demonizzazione. I poliziotti del battaglione che attuarono i massacri e le deportazioni erano esseri umani, esattamente come i pochi che si rifiutarono o si sottrassero a tali compiti. Dovrò perciò riconoscere che, nella stessa situazione, avrei potuto essere un assassino o un disertore [...] se vorrò comprendere o spiegare nel migliore dei modi i loro comportamenti. [...] Senza un tentativo di comprensione dei responsabili in quanto esseri umani, diventa impossibile scrivere non solo questa storia, ma anche qualsiasi storia dei responsabili dell'Olocausto che cerchi di andare oltre la caricatura[30].

Per questa attitudine a utilizzare l'immedesimazione nella ricostruzione storica Browning cita 1'Apologia della storia di Marc Bloch, stesa «poco prima di morire per mano dei nazisti»: «Una parola domina e illumina i nostri studi: comprendere», scriveva lo storico francese. Un'altra frase che viene in mente a proposito di «comprensione» - delle «difficoltà del comprendere», da cui siamo partiti - è quella di Hannah Arendt: «Comprendere il totalitarismo non equivale a condonare alcunché, bensí a riconciliarsi con un mondo in cui questo genere di cose sono semplicemente possibili»[31].

\section{Bibliografia}

- H. Arendt, Comprensione e politica (le difficoltà del comprendere) (1954), in S. Forti (a cura di), Archivio Arendt 2. 1950-1954, trad. di P. Costa, Feltrinelli, Milano 2003 (ed. or. 1994).

- O. Bartov, L'Europa orientale come luogo del genocidio, in M. Cattaruzza, M. Flores, S. Levis Sullam, E. Traverso (a cura di), Storia della Shoah. La crisi dell'Europa, lo sterminio degli ebrei e la memoria del XX secolo, UTET, Torino 2005, pp. 813-849.

- O. Bartov, Anatomy of a Genocide. The Life and Death of a Town called Buczacz, Simon \& Schuster, New York 2018.

- D. Bloxham, A. D. Moses (a cura di), The Oxford Handbook of Genocide Studies, Oxford University Press, Oxford-New York 2010.

- D. Bloxham, Lo sterminio degli ebrei. Un genocidio, trad. di S. Marchesi, Einaudi, Torino 2010 (ed. or. 2009).

- C. Browning, Uomini comuni. Polizia tedesca e «soluzione finale» in Polonia, Einaudi, Torino $1999^{2}$ (ed. or. 1992).

- S. Friedländer, La Germania nazista e gli ebrei. Gli anni della persecuzione (1933-1939), Garzanti, Milano 1998 (ed. or. 1997).

- S. Friedländer, Gli anni dello sterminio. La Germania nazista e gli ebrei (1939-1945), trad. di S. Caraffini, Garzanti, Milano 2009 (ed. or. 2007).

- J.T. Gross, I carnefici della porta accanto, trad. di L. Vanni, Mondadori, Milano 2002 (ed. or. 2001), p. 102.

- R. Hilberg, La distruzione degli ebrei d'Europa, Einaudi, Torino 1995 (ed. or. 1961).

- G. Levi, A proposito di microstoria, in P. Burke (a cura di), La storiografia contemporanea, Laterza, Roma-Bari 1993 (ed. or. 1991), pp. 111-134.

- E. Nolte, Nazionalismo e bolscevismo: la guerra civile europea 1917-1945, Sansoni, Firenze 1988 (ed. or. 1987)

- L. Poliakov, Il nazismo e lo sterminio degli ebrei, Einaudi, Torino 1954.

- T. Snyder, Terre di sangue. L'Europa nella morsa di Hitler e Stalin, trad. di L. Lanza, S. Mancini e P. Vicentini, Rizzoli, Milano 2011 (ed. or. 2010).

- A.V. Sullam Calimani, I nomi dello sterminio. Definizioni di una tragedia, Marietti, Bologna 2018. 
Note:

[1] Per uno sguardo d'assieme cfr. D. Bloxham, A. D. Moses (a cura di), The Oxford Handbook of Genocide Studies, Oxford University Press, Oxford-New York 2010.

[2] D. Bloxham, Lo sterminio degli ebrei. Un genocidio, trad. di S. Marchesi, Einaudi, Torino 2010 (ed. or. 2009).

[3] L. Poliakov, Il nazismo e lo sterminio degli ebrei, Einaudi, Torino 1954.

[4] R. Hilberg, La distruzione degli ebrei d'Europa, Einaudi, Torino 1995 (ed. or. 1961).

[5] Cfr. A.V. Sullam Calimani, I nomi dello sterminio. Definizioni di una tragedia, Marietti, Bologna 2018.

[6] S. Friedländer, Gli anni dello sterminio. La Germania nazista e gli ebrei (1939-1945), trad. di S. Caraffini, Garzanti, Milano 2009 (ed. or. 2007).

[7] S. Friedländer, La Germania nazista e gli ebrei. Gli anni della persecuzione (1933-1939), Garzanti, Milano 1998 (ed. or. 1997).

[8] Friedländer, 2009.

[9] Friedlander, 2009, pp. 10, 9, 13.

[10] J. T. Gross, I carnefici della porta accanto, trad. di L. Vanni, Mondadori, Milano 2002 (ed. or. 2001), p. 102.

[11] Gross, 2002, p. 138.

[12] Gross, 2002, pp. 138-139.

[13] Per una discussione dell'approccio microstorico, cfr. G. Levi, A proposito di microstoria, in P. Burke (a cura di), La storiografia contemporanea, Laterza, Roma-Bari 1993 (ed. or. 1991), pp. 111134.

[14] Gross, 2002, p. 145.

[15] T. Snyder, Terre di sangue. L'Europa nella morsa di Hitler e Stalin, trad. di L. Lanza, S. Mancini e P. Vicentini, Rizzoli, Milano 2011 (ed. or. 2010).

[16] E. Nolte, Nazionalismo e bolscevismo: la guerra civile europea 1917-1945, Sansoni, Firenze 1988 (ed. or. 1987).

[17] Snyder, 2011, pp. 465-466.

[18] Snyder, 2011, p. 450. 
[19] O. Bartov, Anatomy of a Genocide. The Life and Death of a Town called Buczacz, Simon \& Schuster, New York 2018.

[20] Bartov, 2018, p. 36.

[21] Ivi, pp. 67-68, 78.

[22] O. Bartov, L'Europa orientale come luogo del genocidio, in M. Cattaruzza, M. Flores, S. Levis Sullam, E. Traverso (a cura di), Storia della Shoah. La crisi dell'Europa, lo sterminio degli ebrei e la memoria del XX secolo, UTET, Torino 2005, pp. 813-849.

[23] Bartov 2018, p. 133.

[24] Ivi, p. 153.

[25] Ivi, p. 219.

[26] Ivi, pp. 228-229.

[27] C. Browning, Uomini comuni. Polizia tedesca e «soluzione finale» in Polonia, Einaudi, Torino $1999^{2}$ (ed. or. 1992).

[28] Browning, 1999, p. XV (traduzione modificata).

[29] Ivi, p. XIII.

[30] Ivi, p. XVI.

[31] H. Arendt, Comprensione e politica (le difficoltà del comprendere) (1954), in S. Forti (a cura di), Archivio Arendt 2. 1950-1954, trad. di P. Costa, Feltrinelli, Milano 2003 (ed. or. 1994), p. 80. 